\title{
N/O Co-doped Hollow Carbon Nanocapsules-Supported Ge Nanoparticles as Anodes for Excellent Performance Lithium-ion batteries
}

Dacheng Zhang ${ }^{1,2}$, Linlin Wang ${ }^{2, *}$ Qian $\mathrm{Wu}^{2}$, Yujie Yang ${ }^{2}$, and Jingli $X u^{1, *}$

${ }^{1}$ School of Materials Engineering, Shanghai University of Engineering Science, Shanghai 201620, P. R. China.

${ }^{2}$ Institute for Sustainable Energy/College of Science, Shanghai University, 99 Shangda Road, Shanghai 200444, P.R. China.

*E-mail: wlinlin@mail.ustc.edu.cn

Received: 15 June 2021 / Accepted: 10 August 2021 / Published: 10 September 2021

In this work, a novel N/O Co-doped hollow carbon nanocapsule-supported Ge nanoparticles nanocomposite (Ge-N/O-CNCs) was successfully prepared by a facile heating reflux method. N/O codoped hollow carbon nanocapsule-supported Ge nanoparticles not only relieve the agglomeration of Ge nanoparticles and control the volume expansion, but also facilitate the rapid transmission of electrons. In LIBs, the unique Ge-N/O-CNCs nanoarchitecture was used as the anode material. At a high current density of $1 \mathrm{~A} \mathrm{~g}^{-1}$, it remains a stable specific capacity of $448 \mathrm{mAh} \mathrm{g}^{-1}$ remained after 100 cycles. Additionally, even at $3.2 \mathrm{~A} \mathrm{~g}^{-1}$, this material exhibited a high-rate specific capacity of $350 \mathrm{mAh} \mathrm{g}^{-1}$.

Keywords: Ge-N/O-CNCs, LIBs, high capacity, cycling stability

\section{$\underline{\text { FULL TEXT }}$}

(C) 2021 The Authors. Published by ESG (www.electrochemsci.org). This article is an open access article distributed under the terms and conditions of the Creative Commons Attribution license (http://creativecommons.org/licenses/by/4.0/). 